\title{
Ionizing radiation from hydrogen recombination strongly suppresses the lithium scattering signature in the $\mathrm{CMB}$
}

\author{
Eric R. Switzer* and Christopher M. Hirata \\ Department of Physics, Princeton University, Princeton, New Jersey 08544, USA
}

(Received 6 July 2005; published 7 October 2005)

\begin{abstract}
It has been suggested that secondary $\mathrm{CMB}$ anisotropies generated by neutral lithium could open a new observational window into the universe around the redshift $z \sim 400$, and permit a determination of the primordial lithium abundance. The effect is due to resonant scattering in the allowed Li I doublet $\left(2 s^{2} S_{1 / 2}-2 p^{2} P_{1 / 2,3 / 2}\right)$, so its observability depends on the formation history of neutral lithium. Here we show that the ultraviolet photons produced during hydrogen recombination are sufficient to keep lithium in the $\mathrm{Li}$ II ionization stage in the relevant redshift range and suppress the neutral fraction by $\sim 3$ orders of magnitude from previous calculations, making the lithium signature unobservable.
\end{abstract}

DOI: 10.1103/PhysRevD.72.083002

PACS numbers: 98.62.Ra, 26.35.+c, 98.70.Vc, 98.80.Es

\section{INTRODUCTION}

There are few observable traces of structure in the Universe between the cosmic recombination at redshift $z \approx 1100$ and the redshifts $z \leq 7$ accessible to quasar absorption line measurements [1]. Currently our main observational constraints on this redshift range come from the early integrated Sachs-Wolfe (ISW) effect [2] and the large-scale Thomson scattering features in the CMB [3]. These may be supplemented in the future by measurements of the $\mathrm{H}_{\mathrm{I}} 21 \mathrm{~cm}$ hyperfine line [4-8]. However, none of these probes can provide detailed information in the redshift range $200<z<500$ : the free electron abundance is too small to produce significant Thomson scattering, the gas is in thermal equilibrium with the CMB so there is no $21 \mathrm{~cm}$ signal, and the early ISW effect has a blackbody frequency dependence that has no redshift information, making the detection of the effect model-dependent.

The series of papers [9-11] introduces the prospect of using anisotropies in the $\mathrm{CMB}$ generated by scattering in the resonance lines of neutral lithium to study large-scale structure in the redshift range $z \approx 200-500$. Because it involves a resonance, the frequency dependence of the Lis scattering feature can be mapped into a redshift dependence just as is done for the Lyman- $\alpha$ forest and is proposed for high-redshift $21 \mathrm{~cm}$ studies. The frequency dependence deviates strongly from a blackbody, and thus should be distinguishable from the CMB. This idea is also attractive because it provides a potentially clean method of determining the abundance of primordial lithium, before it has been destroyed by nuclear processes in stars. A precision measurement of the lithium abundance from big bang nucleosynthesis (BBN) would be an important consistency check of the cosmological model, and would constrain extensions to standard cosmology such as inhomogeneous BBN [12-14], which tend to increase the ${ }^{7} \mathrm{Li}$ abundance.

*Electronic address: switzer@princeton.edu
The primordial abundance of lithium is extremely small, $X_{\mathrm{Li}} \approx 10^{-10}-10^{-9}$ [15], but the high integrated cross section for excitations of the Lir doublet $2 s^{2} S_{1 / 2}-$ $2 p^{2} P_{1 / 2,3 / 2}$ suggests the possibility of producing a signature within the reach of studies by the Planck, ${ }^{1}$ BLAST, ${ }^{2}$ and $\mathrm{EDGE}^{3}$ experiments - generally experiments with sensitivity around the $300 \mu \mathrm{m}$ band [9]. The Lis doublet has an energy of $1.847 \mathrm{eV}(\lambda=6708 \AA)$, so that the resonant feature would appear to us today at $\lambda=$ $0.6708(1+z) \mu \mathrm{m}$ for scattering at redshift $z$. By comparison, the lowest single-photon excitation for LiII occurs at $61.3 \mathrm{eV}^{4}$ where there are essentially no CMB photons to scatter (and to which the prereionization universe is opaque due to hydrogen and helium photoionization). Hence, an observable lithium signature on the $\mathrm{CMB}$ relies on the formation of neutral lithium after hydrogen recombination.

Previous work on the lithium signature [9-11] emphasizes that with a thermal CMB spectrum, lithium recombines around $z=400$ to form a significant neutral fraction. The neutral lithium gas has an optical depth that is high enough to generate a secondary CMB anisotropy, visible today around $268 \mu \mathrm{m}$. Important to the result is that there are too few ionizing photons in a purely thermal CMB to keep lithium ionized. A separate line of work discusses the nonthermal radiation field generated by hydrogen recombination [16-18], which contains ultraviolet (UV) photons from both Lyman- $\alpha$ production and $2 s \rightarrow 1 s$ two-photon decays. These photons have a maximum energy of $10.1988 \mathrm{eV}$, nearly double the Lis ionization threshold, $\chi_{\mathrm{Li}}=5.3917191 \mathrm{eV}$ [19]. Here, we show that this nonthermal ionizing radiation is sufficient to keep lithium almost completely ionized up to late times, and to hold the optical depth through the resonance below $10^{-4}$ in the

\footnotetext{
${ }^{1}$ http://www.esa.int/science/planck/

${ }^{2}$ http://chile1.physics.upenn.edu/blastpublic/

${ }^{3} \mathrm{http} / / / \mathrm{cfcp}$. uchicago.edu/research/cosmicradiation/edge/

${ }^{4}$ The $61.3 \mathrm{eV}$ excitation of ionized lithium is an intercombination line, and allowed excitations are $>62 \mathrm{eV}$.
} 
standard cosmology. This is significant both from the viewpoint of the secondary lithium anisotropy on the CMB, and more broadly, for lithium recombination and chemistry during this era [20-23].

In this paper, we first calculate the spectral distortion in Sec. II, using the three-level approximation for hydrogen recombination. Section III then discusses the photoionization and recombination rates of lithium in the presence of the spectral distortion. In Sec. IV, we solve the rate equation for lithium's ionization history, and argue that it remains mostly ionized. Section Voutlines the significance this has for a lithium CMB signature. Finally, because this is a nonstandard use of the three-level approximation, we justify it subject to some possible concerns in Sec. VI, and sum up the main results in Sec. VII.

\section{ATOMIC HYDROGEN SPECTRAL DISTORTIONS}

Neutral lithium cannot form if the radiation field contains a sufficient number of photons above the ionization threshold. This makes photoionization dependent on the details of high-energy tail of the photon distribution $(E \geq$ $\left.\chi_{\mathrm{Li}}\right)$, and the photoionization cross section above threshold. The cosmological radiation has nearly a blackbody distribution [24], except for a small population of nonthermal distortion photons from hydrogen recombination in the high-energy tail of the distribution [16-18].

The most important sources of nonthermal radiation during hydrogen recombination are the $\mathrm{H}_{\mathrm{I}}$ two-photon transition $(2 s \rightarrow 1 s)$ and the $\mathrm{H}_{\mathrm{I}}$ Lyman- $\alpha$ transition $(2 p \rightarrow$ $1 s)$. Very few distortion photons are produced in the higher-order Lyman lines because such photons are efficiently destroyed by absorption followed by decay to an excited state of $\mathrm{H}_{\mathrm{I}}$. Also, the Balmer, Paschen, etc. lines of $\mathrm{H}_{\mathrm{I}}$, as well as nearly all of the photons produced by direct recombination to $\mathrm{H}_{\mathrm{I}}$ excited states have energies below $5.39 \mathrm{eV}$, and so cannot ionize Lir. The populations of the $2 s$ and $2 p$ states are expected to vary out of equilibrium at late times. The bearing this has on the formation history of neutral lithium is discussed in Sec. VI.

We will see that the nonthermal photons from $n=2 \rightarrow$ 1 decays of $\mathrm{H}_{\mathrm{I}}$ have an abundance of $10^{-9}$ relative to the peak of the thermal distribution, but exceed the CMB photon population above the ionization threshold of lithium [17], on the high-energy tail of the CMB. The ionizing radiation from these decays must be included in a calculation of lithium's ionization history.

A natural quantity to track is the number of photons per hydrogen, per log-energy. Written in terms of the phase space density $\mathcal{N}$ and specific intensity $J_{\nu}$,

$$
r \equiv\left[\frac{\text { photons }}{\text { hydrogen } \cdot \ln (E)}\right]=\frac{8 \pi E^{3}}{h^{3} c^{3} n_{H}} \mathcal{N}=\frac{4 \pi}{h c n_{H}} J_{\nu},
$$

where $n_{H}$ is the hydrogen number density and $h$ and $c$ are Planck's constant and the speed of light, respectively. This choice also has the advantage that it is conserved along a trajectory, unlike $J_{\nu}$. The photon number densities and baryon number densities both dilute by $(1+z)^{3}$. With these units, the high-energy Wien tail of the blackbody distribution scales as,

$$
r(E, z) \approx \frac{8 \pi E^{3}}{h^{3} c^{3} n_{H}} e^{-E /\left(k T_{r}\right)} .
$$

(After $z \approx 200$, matter and radiation are decoupled, so their thermal histories depart and must be tracked separately as a radiation temperature $T_{r}$, and matter temperature $T_{m}$.) From the integral over the asymptotic distribution, there are $\approx 80$ photons capable of ionizing lithium, per lithium atom at $z=500$, and only $\approx$ $1 \times 10^{-11}$ at $z=300$ from the thermal distribution. Purely thermal CMB radiation is not enough to keep lithium ionized after $z=400$, but the distortion extends to much higher energies.

We find the distortion by calculating the rates of twophoton and Lyman- $\alpha$ decays, and transport the spectrum of photons that is generated from reactions of these rates and the known emission profiles. The method works post-facto because it takes the ionization history of hydrogen and its derivatives, and then calculates the radiation field. Ideally, the evolution of the radiation field would be included in the recombination calculation, self-consistently, rather than computed later. Results from a recombination calculation with a self-consistent radiation field are expected to be different at the percent level because of feedback and other effects [25]. Here, we want to determine whether lithium remains ionized, and do not need anything so nuanced.

Assuming that only the two $n=2$ decays of hydrogen to its ground state contribute to the distortion, all the rate information can be extracted from the total hydrogen recombination rate in the three-level approximation $[16,25]$,

$$
\begin{aligned}
\frac{d x_{e}}{d z}= & \frac{x_{e}^{2} n_{H} \alpha_{H}-\beta_{H}\left(1-x_{e}\right) e^{-E_{L y \alpha} /\left(k T_{m}\right)}}{(1+z) H(z)} \\
& \times \frac{\Lambda_{2 \gamma}+1 /\left(K n_{H}\left(1-x_{e}\right)\right)}{\Lambda_{2 \gamma}+\beta_{H}+1 /\left(K n_{H}\left(1-x_{e}\right)\right)},
\end{aligned}
$$

where $x_{e}$ is the fraction of electrons relative to hydrogen, $\alpha_{H}$ is the case $\mathrm{B}$ recombination rate of hydrogen, $\beta_{H}$ is the effective case $\mathrm{B}$ photoionization rate,

$$
\beta_{H}=\alpha_{H} e^{-\chi_{2 s} /(k T)} \frac{\left(2 \pi m_{e} k T\right)^{3 / 2}}{h^{3}},
$$

$\Lambda_{2 \gamma}$ is the $2 s \rightarrow 1 s$ two-photon decay rate, $\chi_{2 s}$ the ionization energy from the $2 s$ state, and $K=\lambda_{L y \alpha}^{3} /[8 \pi H(z)]$ is the Peebles inverse resonance escape rate from the Lyman- $\alpha$ line. The form of the three-level rate equation is suggestive: the term on the second line is the fraction of excited hydrogen atoms that make the transition $2 p \rightarrow 1 s$ or $2 s \rightarrow 1 s$, relative to the atoms that are photoionized. The term in the numerator displays a two-photon piece and a 
Lyman- $\alpha$ piece, while the denominator also has a contribution from photoionization. This makes it easy to decouple the relative contributions of each process to the overall hydrogen recombination rate: call the fractional rate of change in $x_{e}$ during hydrogen recombination due to two-photon and Lyman- $\alpha$ transitions $\left.\left(d x_{e} / d z\right)\right|_{2 \gamma}$ and $\left.\left(d x_{e} / d z\right)\right|_{L y \alpha}$, respectively.

The solutions to the three-level rate equation and evolution of the matter temperature are evaluated by recfast, developed in Seager et al. [25,26]. We have modified it to return the fractional rates, $\left.\left(d x_{e} / d z\right)\right|_{2 \gamma}$ and $\left.\left(d x_{e} / d z\right)\right|_{L y \alpha}$ as a function of $z$. Throughout, the calculations are based on the cosmology $\Omega_{m}=0.27, \quad \Omega_{b}=0.046, \quad H_{0}=$ $70 \mathrm{~km} \mathrm{~s}{ }^{-1} \mathrm{Mpc}^{-1}, T_{\mathrm{CMB}}=2.728 \mathrm{~K}$, and $Y_{\mathrm{He}}=0.24$.

To understand how photons generated during hydrogen recombination influence the ionization history of lithium, they must be transported to later redshifts. One complication is that resonances in molecules and ions such as $\mathrm{H}_{2}$, $\mathrm{H}_{2}^{+}, \mathrm{H}^{-}, \mathrm{HD}$, and $\mathrm{LiH}$ may have sufficient opacity to remove hydrogen recombination photons in transit as they redshift, softening the flux that ionizes lithium. So long as the optical depth between $5.4 \mathrm{eV}$ and $10.2 \mathrm{eV}$ per $\ln (1+z), n_{H} X_{i} c \sigma H^{-1}$, is much less than unity, these can be neglected. Upper bounds on the abundances for these species are based on the maximum abundances from $[22,23]$. Molecular hydrogen is a particular concern because of its high abundance $\left(X_{\mathrm{H}_{2}}<10^{-5}\right.$ at low $\left.z\right)$. Yet, essentially all of distortion photons are quickly degraded to energies less than $10.2 \mathrm{eV}$ during atomic hydrogen recombination, below the strong Lyman-Werner resonances at $>11.2 \mathrm{eV}$. HD is similar to $\mathrm{H}_{2}$, but has allowed dipole transitions. These are weak (comparing, e.g. $[27,28]$ ) and do not generate significant opacity. Upper bounds on the relevant remaining cross sections are, $\sigma_{\mathrm{H}_{2}^{+}}<7.4 \times$ $10^{-18} \mathrm{~cm}^{2} \quad[29,30], \quad \sigma_{\mathrm{H}^{-}}<4.2 \times 10^{-17} \mathrm{~cm}^{2} \quad[29,31]$, $\sigma_{L i H}<1.2 \times 10^{-18} \mathrm{~cm}^{2}$ [32], resulting in negligible opacities.

Since Thomson scattering does not significantly change the photon energy at the redshifts of interest ${ }^{5}$ and molecular absorption can be neglected, transport amounts to redshifting the emitted distributions. The set of distortion photons around some energy $E$ is the sum over all photons which were produced at an earlier time $\left(z_{\mathrm{em}}\right)$ with energy $E_{\text {em }}$ which could have redshifted to the energy $E$ by

\footnotetext{
${ }^{5}$ When a photon scatters off an electron, it loses a fraction of order $\sim E / m_{e} c^{2}$ of its energy due to recoil, so the condition for recoil from repeated scatterings to be negligible is $\tau \ll m_{e} c^{2} / E$, where $\tau$ is the optical depth. In this case, the distortion photons have energy $E \sim 10 \mathrm{eV}$ and $m_{e} c^{2}=511 \mathrm{keV}$, so $m_{e} c^{2} / E \sim 5 \times$ $10^{4}$. By comparison, distortion photons are first produced around redshift $z \sim 1500$, and the optical depth to this redshift is $\sim 30$. Recoil can be neglected. Likewise, the change in energy due to the Doppler effect over many scatterings is $\Delta E / E \sim$ $\sqrt{\tau} \sqrt{(k T) / m_{e} c^{2}}$, yet $\tau \ll m_{e} c^{2} /(k T)$, so Doppler effects can be neglected.
}

redshift $z$. This defines an integral along the line of sight of the contribution of a particular process to the radiation field,

$$
r_{\text {process }}(E)=\left.\int_{z}^{z_{\mathrm{end}}} d z_{\mathrm{em}} E_{\mathrm{em}} \phi\left(E_{\mathrm{em}}\right) \frac{d x_{e}}{d z}\left(z_{\mathrm{em}}\right)\right|_{\text {process }},
$$

where $\phi\left(E_{\mathrm{em}}\right)$ is the emission profile of the process, evaluated at the energy of the radiation at redshift $z_{\mathrm{em}}$ that will have redshifted down to an energy $E$ by redshift $z$, $E(z)_{\mathrm{em}}=E\left(1+z_{\mathrm{em}}\right) /(1+z)$. The combination of the profile and frequency suggests a new quantity:

$$
\psi\left(E_{\mathrm{em}}\right)=E_{\mathrm{em}} \phi\left(E_{\mathrm{em}}\right)=\left[\frac{\text { photons }}{\text { decay } \cdot \ln \left(E_{\mathrm{em}}\right)}\right] .
$$

The integral Eq. (5) along a line of sight is equivalent to an integral over energy under the change of variables:

$$
E_{\mathrm{em}}=E \frac{\left(1+z_{\mathrm{em}}\right)}{(1+z)} \Rightarrow d z_{\mathrm{em}}=\left(1+z_{\mathrm{em}}\right) \frac{d E_{\mathrm{em}}}{E_{\mathrm{em}}} .
$$

The integral along the line of sight adds contributions from increasingly higher redshifts, with higher emission energies $E_{\mathrm{em}}$. Because the emission profile is the same as time goes on, this is equivalent to adding up different contributions over the profile evaluated at progressively higher energies. The change of variables amounts to converting this indirect way of summing over redshifts into a direct, convenient sum of the profile and rates over energy,

$$
r_{2 \gamma}(E)=\left.\int_{E}^{E_{L y \alpha}}\left(1+z_{\mathrm{em}}\right) \frac{d E^{\prime}}{E^{\prime}} \psi_{2 \gamma}\left(E^{\prime}\right) \frac{d x_{e}}{d z}\left(z_{\mathrm{em}}\left(E^{\prime}\right)\right)\right|_{2 \gamma} .
$$

Here, $z_{\mathrm{em}}$ is the redshift at which light was emitted with energy $E_{\mathrm{em}}$ that will redshift to energy $E$ at some later redshift $z$

$$
z_{\mathrm{em}}(E)=\frac{E_{\mathrm{em}}}{E}(1+z)-1
$$

We use the polynomial fit to the Spitzer \& Greenstein [33] two-photon profile provided in [34], and assume a delta function for the Lyman- $\alpha$ profile. Results from the calculation are shown in Fig. 1. An exciting possibility is that the spectral distortion has sufficient intensity to be observable today. Transporting the distortion to today, its intensity $\nu I_{\nu}$ becomes less than $\sim 10^{-11} \mathrm{erg} \mathrm{cm}^{-2} \mathrm{~s}^{-1} \mathrm{sr}^{-1}$, where the distortion dominates over the CMB for $\lambda<0.13 \mathrm{~mm}$, quickly dropping at smaller wavelengths to $\sim 10^{-14} \mathrm{erg} \mathrm{cm}^{-2} \mathrm{~s}^{-1} \mathrm{sr}^{-1}$. Dust models and observations give an optimistic lower bound on intensity of $\sim 10^{-7} \mathrm{erg} \mathrm{cm}^{-2} \mathrm{~s}^{-1} \mathrm{sr}^{-1}$ in this regime [35], making the distortion extremely difficult to detect today. 


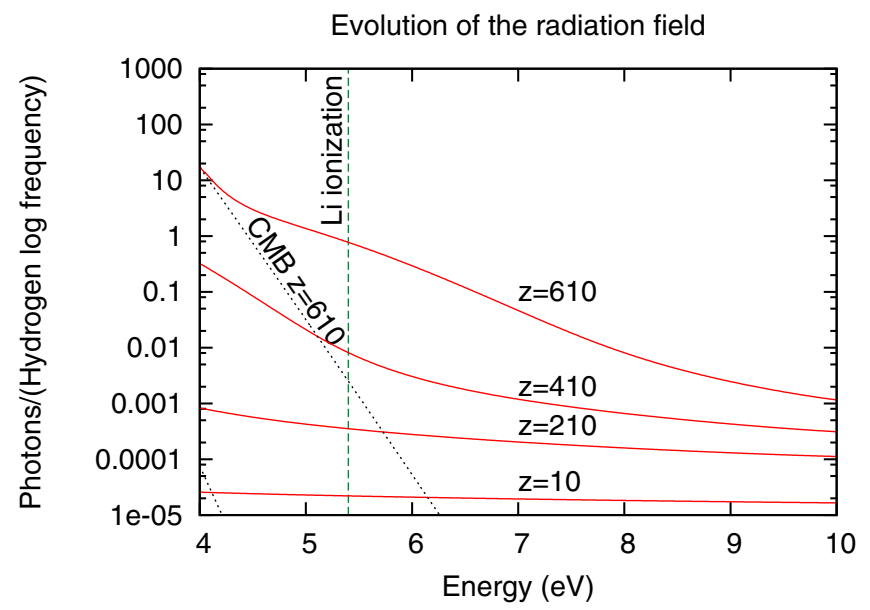

FIG. 1 (color online). The distortion field generated by $n=2$ decays of hydrogen during recombination dwarfs the highenergy tail of the CMB (shown in black dotted line). The ionization energy of lithium is shown to emphasize that even at late times, there is a large number of distortion photons above the ionization energy.

\section{LITHIUM RECOMBINATION AND PHOTOIONIZATION}

The photoionization rate of Lis has a thermal contribution from the CMB and a nonthermal contribution from the integral of the cross section over the distortion field, $\beta_{\mathrm{Li}}=$ $\beta_{\mathrm{CMB}}+\beta_{\mathrm{dist}}$. The thermal photoionization rate is related by the Milne relations to the recombination rate $\alpha_{\mathrm{Li}}$,

$$
\beta_{\mathrm{CMB}}=\frac{g_{e} g_{\mathrm{LiII}}}{g_{\mathrm{LiI}}} \alpha_{\mathrm{Li}}\left(T_{r}\right) \exp \left\{-\frac{\chi_{\mathrm{LiI}}}{k T_{r}}\right\} \frac{\left(2 \pi m_{e} k T_{r}\right)^{3 / 2}}{h^{3}} .
$$

The Milne relations assume Boltzmann-distributed excited states at temperature $T_{r}$. Yet, the distortion photons may increase the population of excited states, increasing the ionization rate from $\mathrm{CMB}$ photons. If this effect is important, it can only increase $\beta_{\mathrm{CMB}}$, and thus reduce the neutral lithium abundance. Ionization by a distortion photon occurs predominantly from the ground state. For the case A $\mathrm{Li}_{\text {II }} \rightarrow \mathrm{Li}$ r recombination rate, $\alpha_{\mathrm{Li}}$, we use a fit from Verner and Ferland [36],

$$
\alpha_{\mathrm{Li}}\left(T_{m}\right)=a\left[\sqrt{\frac{T_{m}}{T_{0}}}\left(1+\sqrt{\frac{T_{m}}{T_{0}}}\right)^{1-b}\left(1+\sqrt{\frac{T_{m}}{T_{1}}}\right)^{1+b}\right]^{-1},
$$

where the coefficients are, $a=1.036 \times 10^{-11} \mathrm{~cm}^{3} \mathrm{~s}^{-1}$, $b=0.3880, \quad T_{0}=1.077 \times 10^{2} \mathrm{~K}, \quad$ and $\quad T_{1}=1.177 \times$ $10^{7} \mathrm{~K}$. The fit is accurate to better than $4 \%$ (with maximum error less than 6\%) [36] between $3 \mathrm{~K}$ and $10^{9} \mathrm{~K}$.

The nonthermal contribution to lithium photoionization from photons emitted during hydrogen recombination is the integral over the cross section,

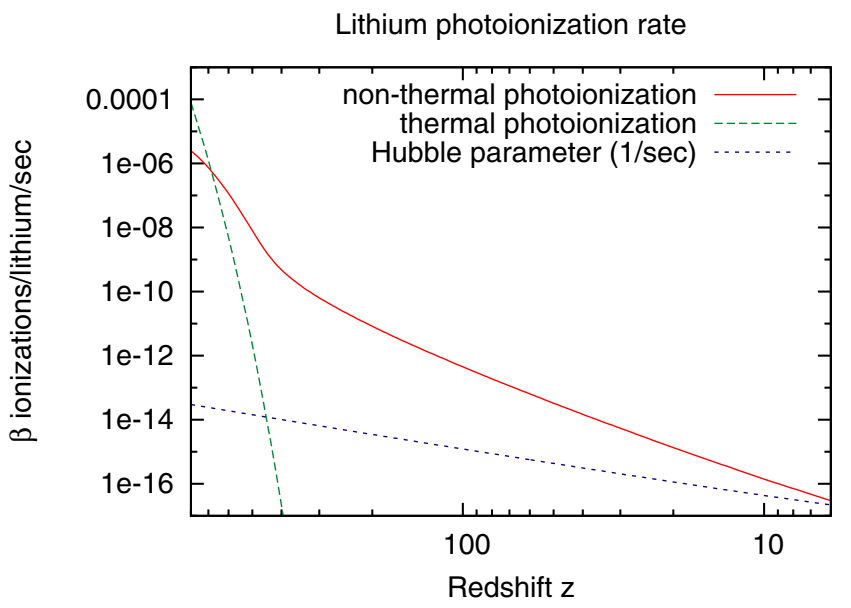

FIG. 2 (color online). The photoionization rate of lithium as a function of redshift, showing the contribution of nonthermal (distortion) photons, beginning to dominate shortly after hydrogen recombination. The nonthermal rate is fast compared to the inverse Hubble time, allowing a steady-state solution.

$$
\beta_{\mathrm{dist}}=\int_{\chi_{\mathrm{Li}_{\mathrm{I}}}}^{E_{L y \alpha}} \frac{d E^{\prime}}{E^{\prime}}\left(r_{2 \gamma}\left(E^{\prime}\right)+r_{L y \alpha}\left(E^{\prime}\right)\right) \sigma_{\mathrm{Li}}\left(E^{\prime}\right) c .
$$

We use a fit to the photoionization cross section for Lir from [37] that has been smoothed over resonances. (There are no resonances in our energy range, so this is not a concern.) The fit is based on low-energy cross sections from the Opacity Project [38] and high-energy cross sections from a Hartree-Dirac-Slater method (though the highenergy cross section is unimportant here because there are no photons above $E_{L y \alpha}=10.2 \mathrm{eV}$ ):

$$
\sigma\left(y=E / E_{0}\right)=\sigma_{0}(y-1)^{2} y^{0.5 P-5.5}\left(1+\sqrt{y / y_{a}}\right)^{-P},
$$

where $\sigma_{0}=1.87723 \times 10^{-6} \mathrm{~cm}^{3} \mathrm{~s}^{-1} / c, P=4.895$, and $y_{a}=15.01$. The results for the nonthermal and thermal contributions to lithium photoionization as a function of redshift are shown in Fig. 2.

\section{IONIZATION HISTORY AND SCALING}

Lithium chemistry and the interaction between lithium ions, lithium hydride, etc. can be very complicated [22], but we will follow Stancil et al. [9] in tracking only photoionization and radiative recombination, the two most dominant reactions,

$$
\mathrm{Li}^{+}+\mathrm{e}^{-} \leftrightarrow \mathrm{Li}+\gamma .
$$

The next leading order reaction is radiative charge transfer [9,39],

$$
\mathrm{Li}+\mathrm{H}^{+} \rightarrow \mathrm{Li}^{+}+\mathrm{H}+\gamma,
$$

which is 2 orders of magnitude slower than Eq. (14) if 
distortion photons are neglected. Photoionization from distortions will only speed up Eq. (14) relative to Eq. (15), making charge transfer less important.

The rate equation describing the evolution of the neutral lithium fraction $f_{\mathrm{Li}}$ based on the recombination $\alpha_{\mathrm{Li}}$ rate and photoionization rate $\beta_{\mathrm{Li}}$ is

$$
\frac{d f_{\mathrm{Li}}}{d z}=-(1+z)^{-1} H(z)^{-1}\left[\alpha_{\mathrm{Li}} n_{e}\left(1-f_{\mathrm{Li}}\right)-\beta_{\mathrm{Li}} f_{\mathrm{Li}}\right] .
$$

The solution of the rate equation Eq. (16) is shown in Fig. 3.

Lithium recombination according to Eq. (16) goes through four major stages:

(1) A Saha stage, where the lithium ionization states are in thermodynamic equilibrium;

(2) A primary steady-state stage, in which the spectral distortion from the bulk of hydrogen recombination at $z \sim 1300$ keeps lithium ionized;

(3) A residual steady-state stage, in which the spectral distortion from $z \sim 1300$ has redshifted to below the Lis ionization threshold, but lithium is kept ionized by UV radiation from residual hydrogen recombinations; and

(4) A freeze-out stage, in which the time scale for all relevant reactions becomes larger than the Hubble time, and $f_{\mathrm{Li}}$ approaches a constant. It is during this stage that the steady-state approximation, Eq. (18), breaks down.

In the real universe, the first stars eventually turn on, increasing the UV background-probably ionizing essen-

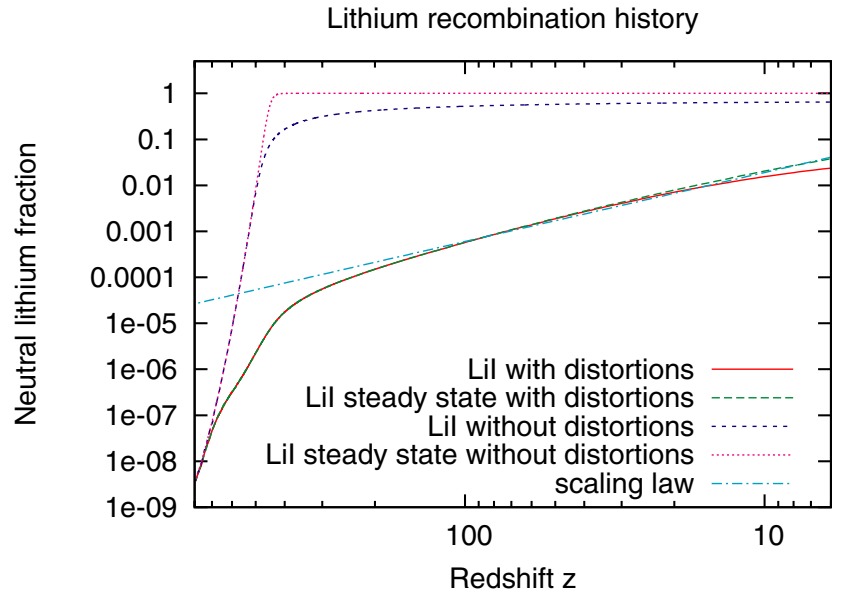

FIG. 3 (color online). Lithium recombination history in the presence of an ionizing flux from the $n=2$ hydrogen recombinations to the ground state. Lithium recombines much more slowly than for a purely thermal CMB, as $(1+z)^{-3 / 2}$, and remains largely ionized up until late times. This reduces the optical depth, and significantly reduces the imprint left on the CMB. tially all of the intergalactic lithium. This occurs too late (i.e. too low $z$ ) to be of interest for the lithium feature in the CMB.

We now discuss each of the four stages in more detail.

\section{A. Saha stage $(z>700)$}

Initially, $\mathrm{Li}$ and $\mathrm{Li}$ I coexist in a Saha equilibrium, where many thermal ionizing photons are present and the distortion is negligible. Note that the neutral fraction during this phase is extremely small, $f_{\mathrm{Li}}<10^{-7}$. The Saha equation in this case is

$$
f_{\mathrm{Li}}=n_{e}\left(\frac{h^{2}}{2 \pi m_{e} k T_{r}}\right)^{3 / 2} e^{\chi_{L i l} /\left(k T_{r}\right)} .
$$

This equation is valid until $z \sim 700$.

\section{B. Primary steady-state stage $(400<z<700)$}

Around $z \sim 700$, enough CMB photons redshift below the Lis ionization threshold so that a small number of distortion photons dominate the $\mathrm{Li}$ photoionization rate. During this second stage, the neutral fraction continues to increase, but it lags far behind the Saha prediction. Although thermodynamic equilibrium no longer applies in this redshift range, the lifetime $\beta_{\mathrm{Li}}^{-1}$ of a neutral lithium atom is still small compared to the Hubble time $H^{-1}$. Thus a "steady state" occurs in which the net Liı production rate $d f_{\mathrm{Li}} / d z$ is much smaller than either the recombination or photoionization rates in Eq. (16). Thus we have

$$
f_{\mathrm{Li}} \approx \alpha_{\mathrm{Li}} n_{e} /\left(\beta_{\mathrm{Li}}+\alpha_{\mathrm{Li}} n_{e}\right) \approx \alpha_{\mathrm{Li}} n_{e} / \beta_{\mathrm{Li}} .
$$

[This steady-state solution for lithium is analogous to the "ionization equilibrium" of $\mathrm{H}_{\mathrm{I}}$ and $\mathrm{H}^{+}$in the Lyman- $\alpha$ forest. In both cases the recombination rate to form the neutral species ( $\mathrm{Li}_{\mathrm{I}}$ or $\mathrm{H}_{\mathrm{I}}$ ) is counterbalanced by photoionization by UV photons from a nonthermal source, which consists of hydrogen recombination radiation in the case of lithium versus stars and quasars in the Lyman- $\alpha$ forest. Neither case represents a true thermodynamic equilibrium because the radiation field is nonthermal.]

\section{Residual steady-state stage $(20<z<400)$}

When hydrogen recombination freezes out at $z \sim 800$, the residual ionization fraction $x_{e} \sim$ few $\times 10^{-4}$ changes very little. The Lyman- $\alpha$ photons produced at $z \sim 800$ are cosmologically redshifted and by $z \sim 400$ are no longer capable of ionizing Liı. Thus one might naively expect that after $z \sim 400$, lithium would fully recombine to Li. As can be seen from Fig. 3, this is not the case: even after hydrogen recombination is "frozen out," there are still a few residual recombinations occurring. These act as a source of UV photons, keeping the neutral lithium fraction small.

During the period of residual recombination, the steadystate solution for the neutral fraction can be simplified into 
a scaling law by making two main assumptions: matter temperature changes adiabatically ( $T \propto a^{-2}$ ) after thermal decoupling at $z \sim 200$, and the relic electron fraction $x_{e}$ is nearly constant after freeze-out. The low temperature limit of the recombination rate coefficient in Eq. (11) for adiabatic matter gives $\alpha_{\mathrm{Li}} \propto T^{-1 / 2} \propto a$. The photoionization rate is then

$$
\beta_{\mathrm{Li}} \propto \alpha_{H} n^{2} x_{e}^{2} t \propto a^{-7 / 2} .
$$

( $a \propto t^{2 / 3}$ for matter domination, $H \propto t^{-1}$. The number density squared scales as $n^{2} \propto a^{-6}$.) Likewise for hydrogen, $\alpha_{H} \propto a$, so that the neutral lithium fraction is given by,

$$
f_{\mathrm{Li}}=\frac{n_{\mathrm{Li}}}{n_{\mathrm{Li}^{+}}+n_{\mathrm{Li}}} \approx \frac{n_{\mathrm{Li}}}{n_{\mathrm{Li}^{+}}}=\frac{\alpha_{\mathrm{Li}}}{\beta_{\mathrm{Li}}} n_{e} \propto \frac{a}{a^{7 / 2}} a^{-3} \propto a^{3 / 2} .
$$

The $f_{\mathrm{Li}} \propto a^{3 / 2}$ scaling law is shown in Fig. 3 and does a remarkably good job of reproducing the full numerical solution in the range from thermal decoupling at $z \sim 200$ to lithium freeze-out at $z \sim 20$.

\section{Freeze-out $(z<20)$}

As the Universe continues to expand, the number of residual recombinations drops. Consequently the UV background becomes fainter, and eventually the lifetime of a lithium atom exceeds the Hubble time, $\beta_{\mathrm{Li}}^{-1} \gtrsim H^{-1}$. Here, the neutral fraction of lithium breaks away from the $a^{3 / 2}$ scaling and approaches a constant (see Fig. 3).

At some point the freeze-out is interrupted by cosmic reionization caused by the first stars and quasars. Current observations [40] indicate that hydrogen was reionized at $z \geq 6$; lithium was presumably ionized even earlier because the prereionization universe was transparent to photons with energies of 5.4-13.6 eV (except in the Lyman lines), which are capable of ionizing lithium. Regardless, this redshift range is not of interest for observations of the lithium resonance line scattering signal: scattering at $z=$ 20 would be observed at a wavelength of $14 \mu \mathrm{m}$, where the CMB radiation is many orders of magnitude fainter than foreground emissions.

\section{SIGNATURE IN THE CMB}

The observability of the lithium signature in the CMB is determined principally by the optical depth through the Lir doublet. As noted in previous work [9-11], the major effect of lithium on small angular scales is to suppress the CMB temperature and polarization anisotropies by a factor of $\exp \left(-\tau_{\mathrm{Li}}\right)$, where $\tau_{\mathrm{Li}}$ is the optical depth through the Lir doublet. This suppression factor depends on the scattering redshift $z_{s}$, which in turn depends on the wavelength of the observation through the usual relation $z_{s}=$ $\lambda_{\text {obs }} /(6708 \AA)-1$. There are also intermediate-scale ef- fects $(\ell \sim 100)$, namely, the Doppler temperature anisotropy due to motion of the gas at the lithium scattering redshift, and the polarization induced by Lis scattering. In the optically thin regime $\tau_{\mathrm{Li}} \ll 1$ of interest here, the magnitude of the effect as measured by, e.g. the change $\Delta C_{\ell}$ in the CMB power spectrum, is proportional to $\tau_{\mathrm{Li}}$. This section is primarily concerned with computing the optical depth $\tau_{\mathrm{Li}}$. We will then argue by scaling from the results of Stancil et al. [9] that the signature is too small to be observed.

The optical depth of neutral lithium in terms of the neutral fraction, neglecting the small fraction occupying the upper states is $[9,25,41]$

$$
\tau_{\mathrm{Li}}\left(\lambda_{\mathrm{obs}}\right)=\sum_{i} \frac{\lambda_{1 i}^{3} A_{i 1}}{8 \pi} \frac{g_{i}}{g_{1}} \frac{f_{\mathrm{Li}}\left(z_{s, i}\right) X_{\mathrm{Li}} n_{H}\left(z_{s, i}\right)}{H\left(z_{s, i}\right)} .
$$

Here the sum extends over all excited levels accessible from the ground level, and the scattering redshifts are given by $z_{s, i}=\lambda_{\text {obs }} / \lambda_{1 i}-1$. Among those, only the $2 p J=1 / 2$ and $J=3 / 2$ excitations at $1.8478 \mathrm{eV}$ contribute for photons at low frequencies (the next allowed excitation is to $3 p^{2} P_{1 / 2,3 / 2}$ at $3.7 \mathrm{eV}$, where dust complicates observations), and each has an Einstein coefficient of $3.72 \times$ $10^{7} s^{-1}$, wavelengths of $\lambda_{1 / 2}=6707.76 \AA$ and $\lambda_{3 / 2}=$ 6707.91 $\AA$, and degeneracies $g_{1 / 2}=2$ and $g_{3 / 2}=4$ $[9,19]$. The degeneracy of the ground level is $g_{1}=2$.

We also need to know the lithium abundance $X_{\mathrm{Li}}$ in order to compute the optical depth. There is some discord about the value of $X_{\mathrm{Li}}$. Recent standard BBN abundance calculations based on the R-matrix method for low-energy nuclear cross sections yield $X_{\mathrm{Li}}=\left(4.15_{-0.45}^{+0.49}\right) \times 10^{-10}$ [42], while observations of low-metallicity stars give $X_{\mathrm{Li}}=$ $\left(1.23_{-0.32}^{+0.68}\right) \times 10^{-10}[43]$. The BBN value of $X_{\mathrm{Li}}=4.15 \times$ $10^{-10}$ is used throughout this analysis. (If the measurement based on stars is correct, the optical depth is correspondingly reduced, $\tau_{\mathrm{Li}} \propto X_{\mathrm{Li}}$.) The optical depth with and without the spectral distortions is shown in Fig. 4.

The optical depths shown here are 3-4 orders of magnitude smaller than those found by Stancil et al. [9] in their Model A (which most closely matches the cosmology used here) in the interesting redshift range $200<z<500$. Instead of a $\sim 20 \%$ change in the CMB power spectrum at $\lambda=268 \mu \mathrm{m}\left(z_{s}=400\right)$, we have $\tau_{\mathrm{Li}} \sim 10^{-5}$ and find a fractional change of the order of $\left|\Delta C_{\ell} / C_{\ell}\right| \sim 2 \times 10^{-5}$. A similar conclusion holds at other redshifts because we find that $\tau_{\mathrm{Li}}$ is always $<10^{-4}$. Thus, with the standard BBN abundance of lithium, the temperature or polarization due to the lithium feature never exceeds about $10^{-4}$ of the primary CMB anisotropy. This is extremely difficult to measure even in principle, and is well beyond the capabilities of current experiments in the relevant wavelength range.

In practice, the lithium signature must also be disentangled from the far infrared background (FIB). 
Lithium Sobolev optical depth

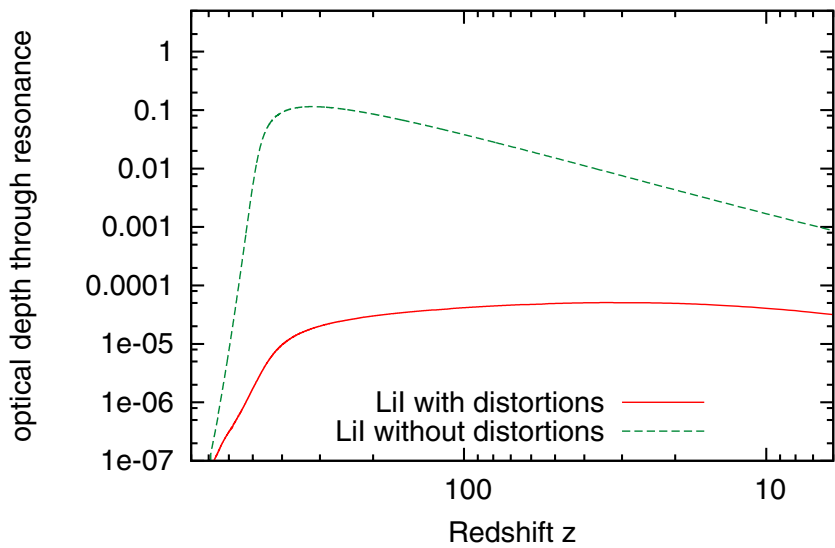

FIG. 4 (color online). A comparison of the optical depth through the resonance of neutral lithium, with and without the ionizing flux from hydrogen recombination.

Zaldarriaga \& Loeb [10] estimate that the FIB fluctuations are comparable to the lithium signal in polarization and dominate over lithium in temperature anisotropy at $z_{s} \sim$ 500 , if $\tau_{\mathrm{Li}}$ is of order unity. At lower $z_{s}$, the FIB-to-CMB ratio increases dramatically. Since we calculate $\tau_{\mathrm{Li}}<$ $10^{-4}$, it follows that the lithium signal should be at most $\sim 10^{-4}$ of the FIB fluctuations. Thus the lithium signal will be undetectable unless both the experimental capabilities improve dramatically, and the FIB fluctuations turn out to be much smaller than predicted or very simple to model.

It is conceivable that the lithium abundance is greater than that predicted by standard BBN, with some inhomogeneous models giving $X_{\mathrm{Li}}$ one to 2 orders of magnitude larger. This is only sufficient to bring $\tau_{\mathrm{Li}}$ up to the $10^{-3}-10^{-2}$ range, which is still much smaller than previous predictions.

\section{SUBTLETIES}

While the three-level approximation provides a convenient way to estimate the spectral distortion from hydrogen recombination, it is a nonstandard application of an approach that was developed largely to calculate the free electron fraction accurately. In this section, we justify its application to the problem of lithium subject to concerns about lithium feedback, i.e. the possibility that Li photoionizations could absorb the spectral distortion (Sec. VI A), $\mathrm{H}_{\mathrm{I}} 2 s$ and $2 p$ falling out of equilibrium at late times, influencing the production of the distortion (Sec. VIB), and the accuracy of case B hydrogen recombination at late times (Sec. VIC).

\section{A. Lithium feedback}

As lithium recombines to the ground state, it absorbs photons from the distortion field. In the most extreme case of this, all of the distortion photons are absorbed by a small number of lithiums, accelerating lithium recombination. We would like to show that this "lithium feedback" is negligible, and that it is realistic to use the distortion field set up by hydrogen recombination alone. This can be shown by checking that the total number of photons absorbed by lithium that could have ionized a Lir atom at redshift $z$ is much less the total number of distortion photons. The number of photons per $\mathrm{H}$ nucleus that are absorbed $\left(X_{\text {removed }}\right)$ is equal to, and bounded by,

$$
\begin{aligned}
X_{\text {removed }}= & \int_{z}^{z_{e}(z)} \frac{X_{\mathrm{Li}}\left(1-f_{\mathrm{Li}}\right) n_{e} \alpha_{\mathrm{Li}}}{H(z)} \frac{d z}{1+z} \\
& +X_{\mathrm{Li}}\left(\left.f_{\mathrm{Li}}\right|_{\text {initial }}-\left.f_{\mathrm{Li}}\right|_{\text {final }}\right) \\
\leq & X_{\mathrm{Li}}\left[\int_{z}^{z_{e}(z)} \frac{n_{e} \alpha_{\mathrm{Li}}}{H(z)} \frac{d z}{1+z}+1\right],
\end{aligned}
$$

where the endpoint of the integral is the maximum redshift where photon from a Lyman- $\alpha$ decay can be created so that it reaches $z$ with enough energy to ionize lithium,

$$
z_{e}(z)=\frac{E_{L y \alpha}}{\chi_{\mathrm{Li}}}(1+z)-1
$$

By comparison, the number of photons above the Lis ionization threshold in the distortion per $\mathrm{H}$ nucleus $\left(X_{\text {field }}\right)$ is

$$
X_{\text {field }}=\int_{\chi_{\mathrm{Li}}}^{E_{L y \alpha}} \frac{d E^{\prime}}{E^{\prime}} r_{\text {distortion }}\left(E^{\prime}, z\right) .
$$

The two integrals, Eqs. (22) and (24), are shown in Fig. 5. The number of photons removed from the distortion is several orders of magnitude less than the total distortion

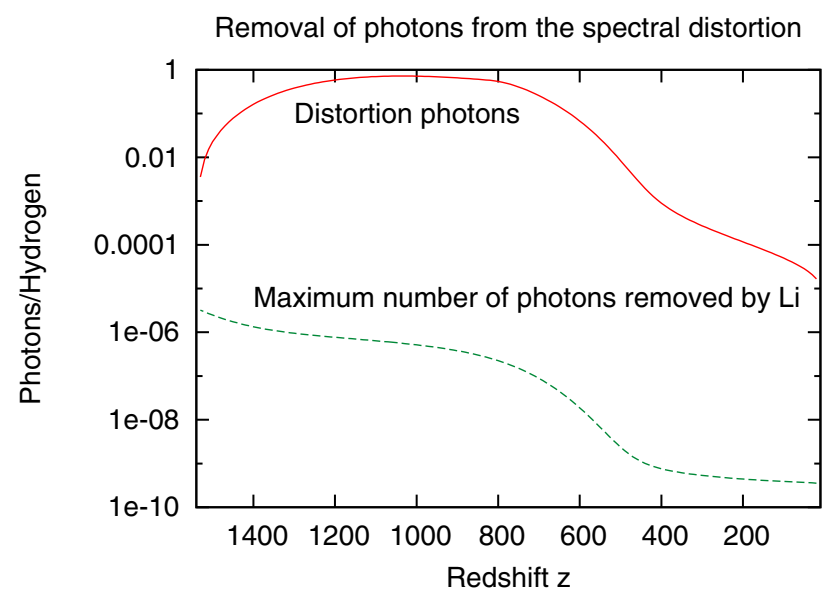

FIG. 5 (color online). The maximum number of photons that lithium recombinations can remove from the distortion population is significantly less than the total distortion population, based on $X_{\mathrm{Li}}=4.15 \times 10^{-10}$. The distortion spectrum is wellapproximated by just those photons which are generated by hydrogen, without lithium feedback. 
population. This confirms that absorption of the distortion photons by lithium can be neglected.

\section{B.2s $-2 p$ nonequilibrium}

The second primary concern is that the spectral distortion derived from rates in the three-level approximation may not be accurate because it assumes that $2 s$ and $2 p$ are in equilibrium in hydrogen. The reactions that keep $2 s$ and $2 p$ in equilibrium involve absorption and emission of Balmer and Paschen line photons. These are expected to slow down not long after hydrogen recombination when the CMB photons redshift to sufficiently low energies that they cannot excite Balmer transitions. This means that $2 s$ and $2 p$ could fall out of equilibrium because the ratio of effective recombination rates $\alpha_{2 p}^{\text {eff }} / \alpha_{2 s}^{\text {eff }}$ differs from the statistical ratio $g_{2 p} / g_{2 s}=3$. This becomes a concern for how well we understand the spectral distortion. If at late times, the majority of the decays are via the twophoton mechanism, then the ionizing flux will not be as hard as when production is a mixture of two-photon and Lyman- $\alpha$, or all Lyman- $\alpha$. Here we look at the worst and best case scenarios where the distortion is produced by only two photon processes, or only Lyman- $\alpha$ processes, shown in Fig. 6. This is a litmus test for whether $2 s$ and $2 p$ being out of equilibrium has any bearing on the conclusion: the lithium ionization history lies somewhere between both of these cases. As can be seen from the figure, even the two extremes do not alter the conclusion that lithium recombination is dramatically inhibited.

The effect of far non-equilibrium distortion production

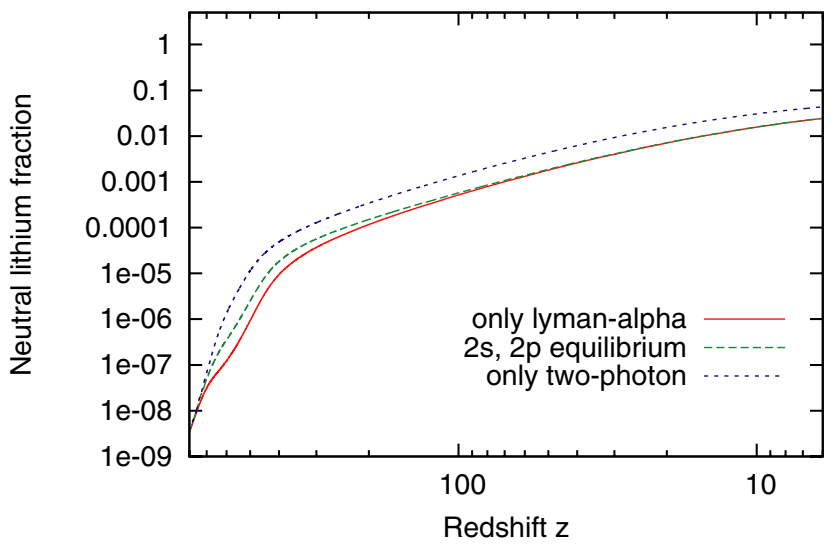

FIG. 6 (color online). For a spectral distortion produced by only Lyman- $\alpha$ decays, the spectral distortion is much harder, and lithium recombines less readily at early times. Alternately, for a distortion produced by only two photon decays, the softer radiation allows lithium to recombine more efficiently. This simulates the effect of hydrogen $2 p$ and $2 s$ falling out of equilibrium in its most extreme form. In both cases, lithium remains largely ionized up to late times.

\section{Accuracy of recombination coefficients}

The third possible concern is that the case B hydrogen recombination coefficients used in recfast from Pequignot et al. [44] will not scale properly below $40 \mathrm{~K}$, where residual hydrogen recombinations can ionize lithium (the coefficients are accurate at high temperatures during the bulk of recombination) [36,45]. The case A hydrogen recombination rates in Verner and Ferland [36] are accurate down to $3 \mathrm{~K}$, with a maximum error of $6 \%$. These can be converted to accurate case $\mathrm{B}$ rates that are similarly accurate down to $3 \mathrm{~K}$ by subtracting recombinations to the $1 s$ level. The fitting form for case $\mathrm{B}$ hydrogen recombination of Pequignot et al. used in recfast departs from this more accurate calculation by less than $\sim 10 \%$ for temperatures above $3 \mathrm{~K}$. The recombination coefficients used in recfast are accurate to several percent over the redshift range in question.

\section{CONCLUSIONS}

We have shown that the nonthermal UV flux from hydrogen recombination is sufficient to keep lithium almost completely ionized throughout the cosmic Dark Ages. Previous studies have only considered the thermal tail of the CMB, which is not enough to ionize lithium at times later than $z \approx 400-500$. The UV flux from hydrogen recombinations dramatically reduces the optical depth through the Lir doublet and the prospect for observing a lithium signal in the CMB. This is unfortunate because the lithium scattering signal, had it been detectable, would have been a valuable cosmological probe in the $200<z<$ 500 redshift range and would have improved our understanding of BBN physics by permitting a clean measurement of the primordial lithium abundance.

UV radiation from hydrogen recombination may also be important for the production of molecules such as $\mathrm{H}_{2}$, $\mathrm{LiH}$, and $\mathrm{HD}$ because some of the intermediate products, catalysts, and final states can be destroyed by UV radiation. $\mathrm{H}_{2}$ and $\mathrm{HD}$ have been suggested as coolants for gas clouds in the early universe which could be important for the formation of the first baryonic structures. Cooling processes will also be crucial in our understanding of upcoming high-redshift $21 \mathrm{~cm}$ surveys, both directly if an $\mathrm{H}_{\mathrm{I}}$ signal from minihaloes is observed [46], and indirectly because cooling affects the formation of UV sources such as stars. These results highlight the importance of developing a code to track the radiation field during recombination, and we hope to discuss a new algorithm for this in a future paper.

\section{ACKNOWLEDGMENTS}

C.H. acknowledges the support of NASA Grant No. NGT5-50383. We acknowledge useful discussions with Uroš Seljak and Avi Loeb. 
[1] X. Fan, V. K. Narayanan, R.H. Lupton, M. A. Strauss, G. R. Knapp, R. H. Becker, R. L. White, L. Pentericci, S. K. Leggett, Z. Haiman et al., Astrophys. J. 122, 2833 (2001).

[2] W. Hu and N. Sugiyama, Phys. Rev. D 51, 2599 (1995).

[3] A. Kogut, D. N. Spergel, C. Barnes, C. L. Bennett, M. Halpern, G. Hinshaw, N. Jarosik, M. Limon, S. S. Meyer, L. Page et al., Astrophys. J. Suppl. Ser. 148, 161 (2003).

[4] P. Madau, A. Meiksin, and M. J. Rees, Astrophys. J. 475, 429 (1997).

[5] M. Zaldarriaga, S. R. Furlanetto, and L. Hernquist, Astrophys. J. 608, 622 (2004).

[6] S. R. Furlanetto, M. Zaldarriaga, and L. Hernquist, Astrophys. J. 613, 16 (2004).

[7] A. Loeb and M. Zaldarriaga, Phys. Rev. Lett. 92, 211301 (2004).

[8] N. Y. Gnedin and P. A. Shaver, Astrophys. J. 608, 611 (2004).

[9] P. C. Stancil, A. Loeb, M. Zaldarriaga, A. Dalgarno, and S. Lepp, Astrophys. J. 580, 29 (2002).

[10] M. Zaldarriaga and A. Loeb, Astrophys. J. 564, 52 (2002).

[11] A. Loeb, Astrophys. J. Lett. 555, L1 (2001).

[12] J.H. Applegate and C. J. Hogan, Phys. Rev. D 31, 3037 (1985).

[13] K. E. Sale and G. J. Mathews, Astrophys. J. Lett. 309, L1 (1986).

[14] G. J. Mathews, C. R. Alcock, and G. M. Fuller, Astrophys. J. 349, 449 (1990).

[15] S. Burles, K. M. Nollett, and M. S. Turner, Phys. Rev. D 63, 063512 (2001).

[16] P. J. E. Peebles, Astrophys. J. 153, 1 (1968).

[17] P. Boschan and P. Biltzinger, Astron. Astrophys. 336, 1 (1998).

[18] G. B. Rybicki and I. P. dell'Antonio, in ASP Conf. Ser. 51: Observational Cosmology, 1993 (unpublished), p. 548.

[19] W. C. Martin, J. Reader, and W. L. Wiese, Atomic Data for X-Ray Astronomy, 25th meeting of the IAU, Joint Discussion 17, 2003, Sydney, Australia, 2003, Vol. 17 (unpublished).

[20] P. C. Stancil, S. Lepp, and A. Dalgarno, Astrophys. J. 458, 401 (1996).

[21] A. Dalgarno and S. Lepp, in IAU Symp. 120: Astrochemistry, 1987 (unpublished), p. 109.

[22] D. Galli and F. Palla, Astron. Astrophys. 335, 403 (1998).
[23] S. Lepp, P. C. Stancil, and A. Dalgarno, J. Phys. B 35, R57 (2002).

[24] D. J. Fixsen, Astrophys. J. Lett. 594, L67 (2003).

[25] S. Seager, D. D. Sasselov, and D. Scott, Astrophys. J. Suppl. Ser. 128, 407 (2000).

[26] S. Seager, D. D. Sasselov, and D. Scott, Astrophys. J. Lett. 523, L1 (1999).

[27] P.C. Stancil and A. Dalgarno, Astrophys. J. 490, 76 (1997).

[28] P.C. Stancil and A. Dalgarno, Astrophys. J. 479, 543 (1997).

[29] M. Tegmark, J. Silk, M. J. Rees, A. Blanchard, T. Abel, and F. Palla, Astrophys. J. 474, 1 (1997).

[30] P. C. Stancil, Astrophys. J. 430, 360 (1994).

[31] A. W. Wishart, Mon. Not. R. Astron. Soc. 187, 59P (1979).

[32] K. Kirby and A. Dalgarno, Astrophys. J. 224, 444 (1978).

[33] L. J. Spitzer and J. L. Greenstein, Astrophys. J. 114, 407 (1951).

[34] M. A. Dopita and R.S. Sutherland, Astrophysics of the Diffuse Universe (Springer, New York, 2003), ISBN .

[35] R. Salvaterra and C. Burigana, Mon. Not. R. Astron. Soc. 336, 592 (2002).

[36] D. A. Verner and G. J. Ferland, Astrophys. J. Suppl. Ser. 103, 467 (1996).

[37] D. A. Verner, G. J. Ferland, K. T. Korista, and D. G. Yakovlev, Astrophys. J. 465, 487 (1996).

[38] W. Cunto, C. Mendoza, F. Ochsenbein, and C. J. Zeippen, Astron. Astrophys. 275, L5 (1993).

[39] P. C. Stancil and B. Zygelman, Astrophys. J. 472, 102 (1996).

[40] X. Fan, V. K. Narayanan, M. A. Strauss, R. L. White, R. H. Becker, L. Pentericci, and H. Rix, Astron. J. 123, 1247 (2002).

[41] E. Bougleux and D. Galli, Mon. Not. R. Astron. Soc. 288, 638 (1997).

[42] A. Coc, C. Angulo, E. Vangioni-Flam, P. Descouvemont, and A. Adahchour, Nucl. Phys. A752, 522 (2005).

[43] S. G. Ryan, T. C. Beers, K. A. Olive, B. D. Fields, and J. E. Norris, Astrophys. J. Lett. 530, L57 (2000).

[44] D. Pequignot, P. Petitjean, and C. Boisson, Astron. Astrophys. 251, 680 (1991).

[45] D. G. Hummer, Mon. Not. R. Astron. Soc. 268, 109 (1994).

[46] I. T. Iliev, E. Scannapieco, and P. R. Shapiro, Astrophys. J. 624, 491 (2005). 